\title{
Achieving Biosensing at Attomolar Concentrations of Cardiac Troponin $T$ in Human Biofluids by Developing a Label-Free Nanoplasmonic Analytical Assay
}

\author{
Thakshila Liyanage, ${ }^{1}$ Andeep Sangha, ${ }^{1}$ and Rajesh Sardar ${ }^{1,2, *}$ \\ ${ }^{1}$ Department of Chemistry and Chemical Biology, Indiana University-Purdue University Indianapolis, \\ 402 N. Blackford Street, Indianapolis, Indiana 46202, United States \\ ${ }^{2}$ Integrated Nanosystems Development Institute, Indiana University-Purdue University Indianapolis, \\ 402 N. Blackford Street, Indianapolis, Indiana 46202, United States
}

Email: rsardar@iupui.edu

\begin{abstract}
Acute myocardial infarction (heart attack) is the fifth leading cause of death in the United States (Dariush et al. Circulation 2015, 131, e29-e322). This highlights the needs for early, rapid, and sensitive detection of its occurrence and severity through assaying cardiac biomarkers in human fluids. Herein we report chip-based fabrication of the first label-free, nanoplasmonic biosensor to assay cardiac Troponin T (cTnT) in human biofluids (plasma, serum, and urine) with high specificity. The sensing mechanism is based on the adsorption model that measures the localized surface plasmon resonance (LSPR) wavelength shift of anti-cTnT functionalized gold triangular nanoprisms (Au TNPs) induced by change of their local dielectric environment upon binding of cTnT. We demonstrate that controlled manipulation of sensing volume and decay length of Au TNPs together with the appropriate surface functionalization and immobilization of anti-cTnT onto TNPs allows us to achieve a limit of detection (LOD) of our cTnT assay at attomolar concentration ( $15 \mathrm{aM})$ in human plasma. This LOD is at least 50 fold more sensitive than that of other label-free techniques. Furthermore, we demonstrate excellent sensitivity of our sensors in human serum and urine. Importantly, the strategy of our chip-based fabrication is extremely reproducible. We believe our powerful analytical tool for detection of cTnT directly in human biofluids using this highly reproducible, label-free LSPR sensor will have great potential for early diagnosis of heart attack and thus increase patients' survival rate.
\end{abstract}

This is the author's manuscript of the article published in final edited form as:

Liyanage, T., Sangha, A., Sardar, R., Brondani, D., Piovesan, J. V., Westphal, E., ... \& Vieira, I. C. (2017). Achieving biosensing at attomolar concentrations of cardiac troponin $\mathrm{T}$ in human biofluids by developing a label-free nanoplasmonic analytical assay. Analyst, $142,2442-2450$. https://doi.org/10.1039/c7an00430c 

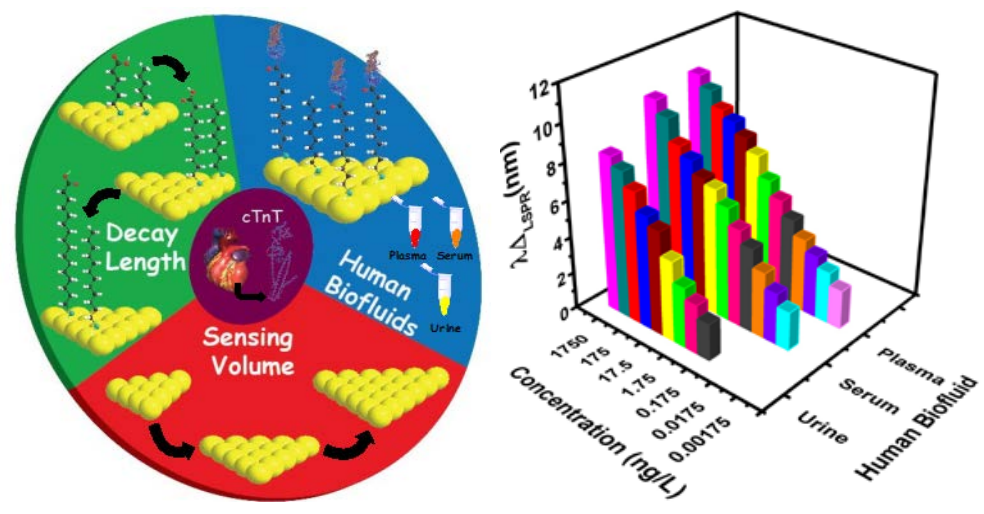

Keywords: localized surface plasmon resonance, nanoprisms, biosensors, cardiac Troponin $\mathrm{T}$, sensing volume, decay length, human biofluids

One sentence synopsis: Nanoplasmonic-based highly reproducible and ultrasensitive analytical sensor was fabricated to quantify cardiac Troponin $\mathrm{T}$ at attomole concentration with high selectivity.

\section{INTRODUCTION}

Over the last 20 years, the field of analytical chemistry has witnessed tremendous progress in label-free quantification of biomolecules in complex biological media utilizing the unique optical properties of noble metal ( $\mathrm{Ag}$ and $\mathrm{Au}$ ) nanoparticles. ${ }^{1-13}$ These nanoparticles display unique localized surface plasmon resonance (LSPR) properties ${ }^{1,4,6,13}$ originating from the coherent oscillation of conduction electrons on their surface when excited by incident photons. In addition to size and shape of a nanoparticle, the local dielectric environment modulates its LSPR frequency. ${ }^{14}$ In this context, ultrasensitive and novel biosensors can be developed by characterizing the LSPR response (extinction or absorption peak position and intensity) of nanoparticles as a function of the changes in their local dielectric environment. ${ }^{6,15-17}$ In this article, we report the first quantification of cardiac Troponin-T (cTnT) in diverse human biofluids (plasma, serum, and urine) utilizing a LSPR biosensor. Our chip-based LSPR cTnT biosensor provides a limit of detection (LOD) as low as $507 \mathrm{fg} / \mathrm{L}$, which is $10^{4}$ fold more sensitive than the commercial Elecys troponin T electrolumi- 
nescense immunoassay (ECLIA, $4^{\text {th }}$ generation, Roche Diagnostic) ${ }^{18}$ and at least 50 fold lower than other label-free analytical techniques. ${ }^{19,20}$

The initial LSPR biosensor work by the Chilkoti21 and Van Duyne ${ }^{13}$ groups demonstrated that in chip-based sensors, fabrication requires three important steps: (i) Immobilization of chemicallysynthesized/lithographically-fabricated nanoparticles onto suitable transparent solid substrates. (ii) Functionalization of the nanoparticle surface with analyte-specific receptors (bio-recognition molecules) that results in fabrication of LSPR biosensors. (3) Assay of analytes through incubation of biosensors in which analyte binding to receptors alters the local refractive index (R.I.) of the nanoparticles inducing a change in the LSPR properties. This change could be either in the form of a variation in the peak intensity or as a shift in the LSPR peak position $\left(\Delta \lambda_{\text {LSPR }}\right)^{4,13}$ This LSPRbased quantification of biomolecules closely resembles the analytical models demonstrated by Arnold and coworkers ${ }^{22}$ and Stenberg et al. $^{23}$ for quantification of surface-bound proteins.

In the context of the LSPR-based bioanalytical sensing mechanism, there are several nanoscale structural parameters that control the overall performance of the sensor. The size and shape of the nanoparticles are critical factors in maximizing the sensor response. More precisely, these structural parameters control the electromagnetic (EM)-field enhancement, ${ }^{6,8,24}$ sensing volume, and decay length that together determine the sensitivity. Moreover, as mentioned above, design of every LSPR-based biosensor requires functionalization of the nanoparticle surface with a receptor. ${ }^{4,8,25}$ Thus linker length, which positions the analyte-receptor complex, and the density of receptors are important. Therefore, to prepare LSPR biosensors with unique function and applications, a quantitative understanding of the manner in which sensing volume, decay length, and surface ligand structural parameters influence nanoparticle optical response during the assay is needed. Note that glass substrate is commonly used in chip-based fabrication of biosensors.

In this article, we study the effects of sensing volume of gold triangular nanoprisms (Au TNPs) on ultrasensitive detection of cTnT as a model analyte. Our chip-based fabrication of the nanoplasmonic cTnT biosensor was performed according to Fig. 1. Our experimental data show that the decay length of Au TNPs that is controlled by varying the number of methylene unit of alkylthiols, plays a critical role in achieving high sensitivity with reduced non-specificity in undiluted human plasma. We also investigated the influence of receptor (anti-cTnT) concentration attached to the 
surface of Au TNPs to obtain maximum sensitivity without compromising the selectivity. Taken together, we determine that a biosensor constructed with (i) an 42 nm edge-length Au TNP, (ii) $1.7 \mathrm{~nm}$ distance between $\mathrm{Au}$ TNP and anti-cTnT, and (iii) an 8:2 ratio of receptor-to-spacer provides LODs of 7.2, 14.5, 21.9 aM in phosphate buffer saline (PBS), human plasma, and human serum, respectively. Furthermore, we also show that our chip-based fabrication methodology is highly reproducible in which biosensors fabricated six months apart (batch-to-batch variation) produced nearly identical sensitivity. Owing to the enormous importance of cTnT in human health where $>10 \mathrm{ng} / \mathrm{L}$ level is considered to be high risk for Myocardial Infarction (MCI), ${ }^{26-35}$ we expect that our simple, cost-effective, and ultrasensitive assay in real human biofluids will be capable of detecting the potentially minute change in cTnT concentrations in symptom-free (chest, back, or jaw pain, and stable coronary artery disease) and poorly differentiated cases and allow interpretation to prevent heart attack by early diagnosis, thereby greatly improving patient survival. ${ }^{36}$ Finally, our determination of the most influential nanoscale structural parameters and our methodology to control surface functionalization and immobilization of receptors will provide experimental benchmarks for the fabrication of ultrasensitive biosensors to quantitatively study a variety of important protein-protein interactions.

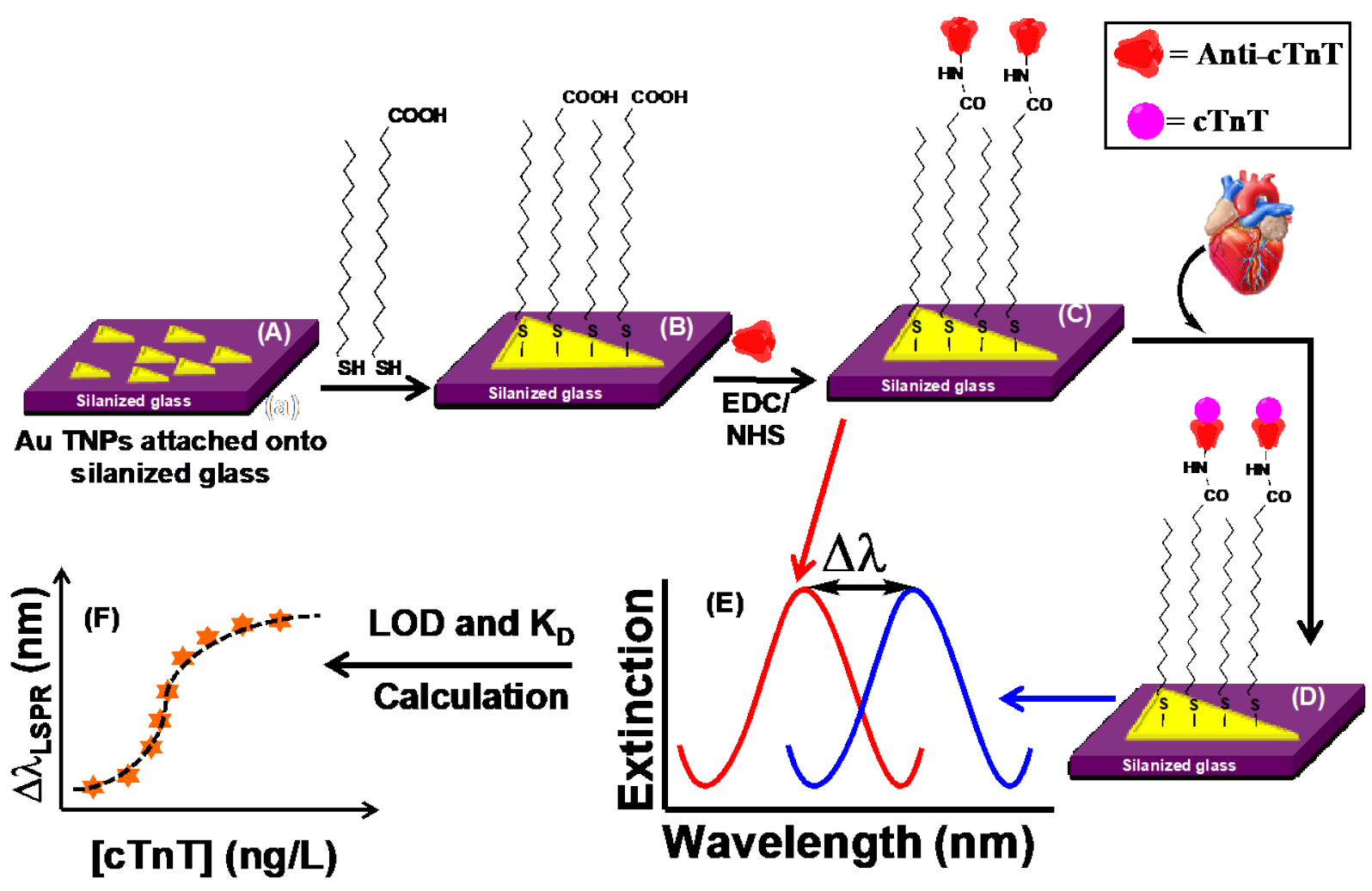


Fig. 1: Design of a chip-based format LSPR cTnT biosensor. (A) Au TNPs attached onto silanized glass, (B) after being functionalized with a 1:1 mole ratio of 1-dodecanethiol and 16-mercaptohexadecanoic acid, (C) further functionalization with anti-cTnT through EDC/NHS amide coupling to complete the nanosensor, (D) detection of cTnT upon binding to anti-cTnT on sensor surface, (E) representation of nanosensor absorption maxima $\left(\lambda_{\mathrm{LSPR}}\right)$ peak shift before and after binding of cTnT, and (F) relationship between $\Delta \lambda_{\mathrm{LSPR}}$ and cTnT concentration to calculate the LOD and $K_{D}$. For simplicity, only one Au TNP is shown in the functionalization steps. The image is not to scale.

\section{EXPERIMENTAL SECTION}

Materials. Chloro(triethylphosphine) gold (I) (Et3PAuCl, 97\%), poly(methylhydrosiloxane) (PMHS, $\mathrm{Mn}=1700-3300)$, trioctylamine $(\mathrm{TOA}, 98 \%)$, ACS grade acetonitrile $\left(\mathrm{CH}_{3} \mathrm{CN}, 99.9 \%\right)$, methanol (99.8\%), N-hydroxysulfosuccinimide solution (NHS), 1-ethyl-3-(3-dimethylamino) propyl carbodiimide (EDC), human plasma, human serum, and all alkylthiols were purchased from Sigma-Aldrich and were used without further purification. Human urine was purchased from (UTAK Laboratories). Human cTnT and sheep anti-cTnT were obtained from Protein-Specialists and Fisher Scientific, respectively. 3-Mercaptopropyl)-triethoxysilane (MPTES, 94\%) was purchased from Alfa Aesar. All organic solvents, reagents for PBS buffer $(\mathrm{pH}=7.4)$ preparation, RBS35 detergent, and the glass coverslips (Cat. No. 12548C) were purchased from Thermo Fisher Scientific. Nanopure water was used for PBS buffer preparation and for all cleaning purposes.

\section{Preparation of Chip-Based Format Biosensors and Quantification of cTnT in Biofluids. The} Electronic Supplementary Information file provides details describing the chemical attachment of $\mathrm{Au}$ TNPs onto glass coverslips. To understand the effects of nanoscale structural parameters on sensor performance, coverslips containing Au TNPs were incubated in an ethanolic solution of thiols to prepare self-assembled monolayers (SAMs) as follows: (a) For sensing volume optimization, 16-mercaptohexadecanoic acid (MHDA) and 1-dodecanethiol (DDT) each of $1.0 \mathrm{mM}$ concentration were used. (b) For decay length optimization in addition to the MHDA/DDT SAM, two additional SAMs were prepared using 6-mercaptohexanoic acid (MHNA) and 1-hexanethiol (HT), or 11-mercaptoundecanoic acid (MUDA) and 1-nonanethiol (NT) each using $1.0 \mathrm{mM}$ concentration. (c) For optimization of cTnT binding sites, we used MUDA/NT SAMs of varying mole ratios. After the SAM preparation, coverslips were washed with plenty of ethanol and then extinction spectra were collected. Next, SAM-modified Au TNPs were incubated in a PBS buffer solution containing 
EDC/NHS ( $0.2 \mathrm{M}$ each) for $2 \mathrm{~h}$ to activate the carboxylic group. The coverslips were then placed into a solution containing $10.0 \mu \mathrm{g} / \mathrm{L}$ of sheep-anti-TnT, incubated overnight at room temperature, then washed with a copious amount of PBS buffer, and stored in buffer for further use. This produced our chip-based format LSPR biosensors. The biosensors were then used to determine the LODs in PBS buffer and various biological fluids (pure human plasma and serum, and 50\% human urine) by incubating for 8-12 $\mathrm{h}$ in different concentrations of $\mathrm{cTnT}$, which were prepared through serial dilution. An $\sim 2.5 \mathrm{~mL}$ of cTnT solution is required to incubate 5 LSPR cTnT biosensors. 

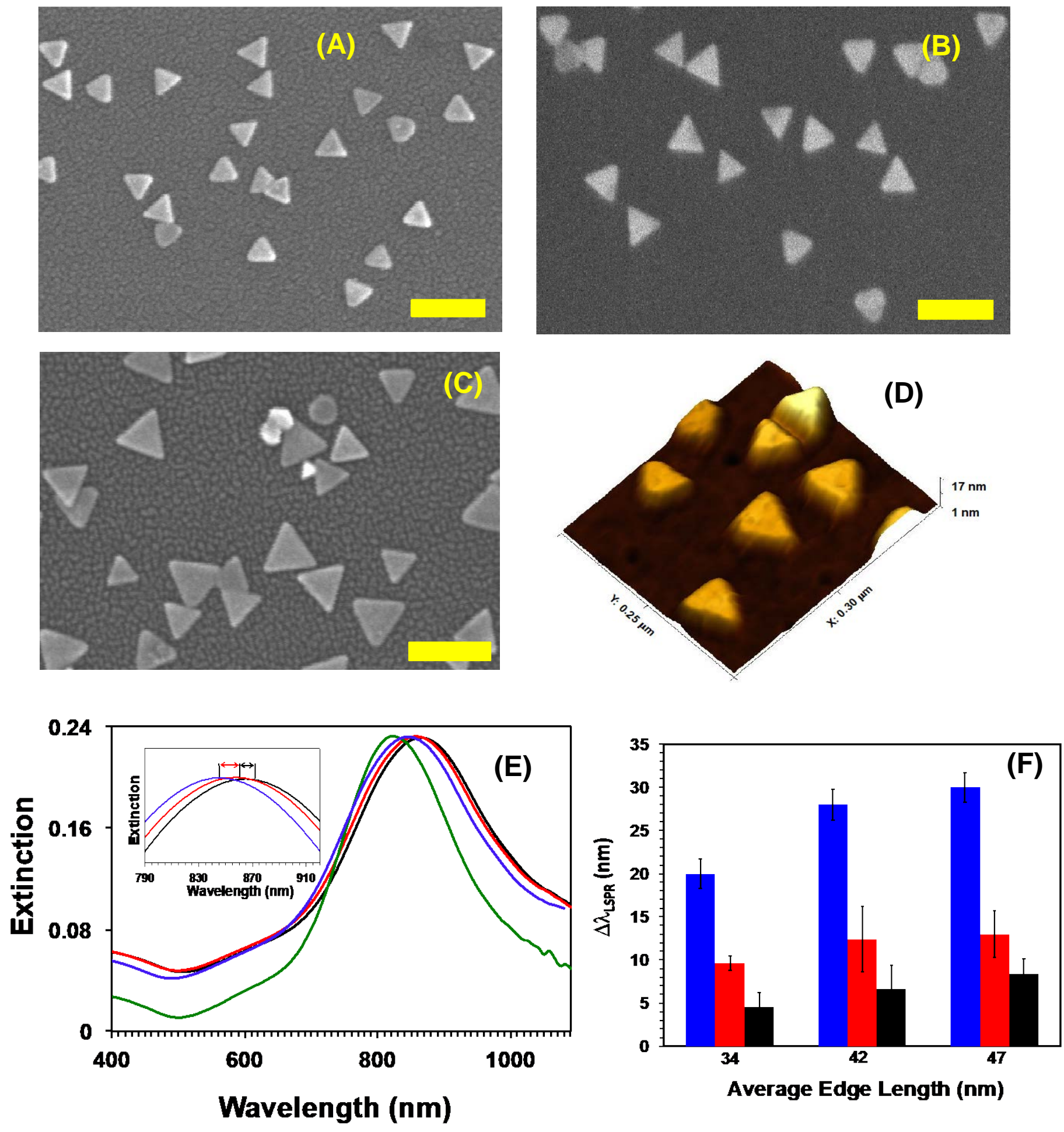

Fig. 2: Microscopy and spectroscopy characterization of chip-based LSPR cTnT biosensors. Scanning electron microscopy (SEM) image of $34 \mathrm{~nm}$ (A), $42 \mathrm{~nm}(\mathrm{~B})$, and $47 \mathrm{~nm}$ (C) edge-length Au TNPs. The scale bars are $100 \mathrm{~nm}$. (D) Atomic force microscopy image of $42 \mathrm{~nm}$ edge-length Au TNPs. (E) UV-visible extinction spectra of $42 \mathrm{~nm}$ edge-length Au TNPs attached onto silanized glass substrate (green, $\lambda_{\mathrm{LSPR}}=825 \mathrm{~nm}$ ) and after MHDA/DDT SAM formation (blue, $\lambda_{\mathrm{LSPR}}=852 \mathrm{~nm}$ ), after attachment of anti-cTnT through EDC $/$ NHC coupling $\left(\mathrm{red}, \lambda_{\mathrm{LSPR}}=864 \mathrm{~nm}\right.$ ), and after incubation in $10 \mathrm{nM}$ cTnT solution $\left(\right.$ black, $\lambda_{\mathrm{LSPR}}=871$ 
$\mathrm{nm})$. Inset shows expanded region of the extinction spectra. All extinction spectra were collected in PBS buffer in order to avoid the effects of bulk refractive index (R.I.) caused by surrounding media. The red and black double-headed arrows represent the difference in wavelength before and after anti-cTnT $\left(\Delta \lambda_{\mathrm{LSPR}}=12\right.$ $\mathrm{nm})$, and after cTnT adsorption $\left(\Delta \lambda_{\mathrm{LSPR}}=7 \mathrm{~nm}\right)$, respectively. (F) Average $\Delta \lambda_{\mathrm{LSPR}}$ values for three different edge-lengths Au TNPs after MHDA/DDT SAM formation (blue bars), anti-cTnT attachment (red bars), and incubation in $10 \mathrm{nM}$ cTnT solution (black bars).

\section{RESULTS AND DISCUSSION}

Optimization of Sensing Volume of Chip-Based LSPR cTnT Biosensor. We selected Au TNPs to fabricate our LSPR biosensors for their unique structural and optical properties as delineated below:

(i) In order use the LSPR biosensors as analytical tools it is important to perform measurements under normal atmosphere and in actual biofluids. Non-noble metal nanoparticles such as copper and aluminum are susceptible to corrosion under these conditions. ${ }^{37}$ Although silver nanoparticles display very high LSPR sensitivity, they undergo fast photooxidation in air under physiological conditions, and thus optical measurements are generally performed under more inert conditions.

(ii) TNPs demonstrate strong EM-field enhancement at their sharp tips and edges ${ }^{38-40}$ and are thus capable of providing higher sensitivity than spherical gold nanoparticles or nanorods. ${ }^{6}$ Although nanostars could provide better sensitivity ${ }^{1}$ due to the presence of multiple arms with sharp tips in their structure, controlling the overall size and arm length is extremely difficult. Therefore, large batch-to-batch variations in their structure could result in large deviations in analytical measurements. A few other geometries of $\mathrm{Au}$ such as nanorice, nanorings, and nanoshells provide high LSPR sensitivity but, they display $\lambda_{\text {LSPR }}>900 \mathrm{~nm} .^{6}$ This spectral region is not ideal for biosensing applications in part because of high background absorption and scattering from endogeneous chromophores in biofluids (e.g., plasma, serum, urine). In this context, the spectral region between $700-900 \mathrm{~nm}$ is the most suitable for LSPR-based biosensing and we can utilize this by controlling the edge-length of the Au TNPs. ${ }^{42}$

(iii) TNPs display larger sensing volume than spherical/disk-shaped nanoparticles. Furthermore, this volume can be controlled by varying their edge-lengths while maintaining their thickness, and longer edge-length TNPs provide larger LSPR sensing volume (Vs). ${ }^{13}$ The Vs is defined as the fixed volume surrounding a TNP that contains $95 \%$ of its sensitivity. ${ }^{43}$ 
We first chemically synthesized 34,42 , and $47 \mathrm{~nm}$ average edge-length $\mathrm{Au}$ TNPs that displayed LSPR dipole extinction peak ( $\lambda_{\text {LSPR }}$ ) at 750, 800, and $820 \mathrm{~nm}$, respectively (Fig. S1), using our published procedure. ${ }^{15,44}$ Fig. 2A-C show representative SEM images of three different edge-length $\mathrm{Au}$ TNPs bound onto glass coverslips. Based on our published work, these Au TNPs are found to be nearly same $( \pm 1.5 \mathrm{~nm})$ thickness. ${ }^{44-47}$ A representative atomic force microscopy image is shown in Fig. 2D. We investigated three nanoscale structural parameters to maximize the biosensor response, and further evaluated the sensor performance in quantifying cTnT in complex biofluids. Fig. 2E illustrates the step-wise $\lambda_{\text {LSPR }}$ shifts of $42 \mathrm{~nm}$ edge-length $\mathrm{Au}$ TNPs at different functionalization steps. Firstly, incubation of glass coverslip containing Au TNPs in mixed MHDA/DDT solution (1:1 ratio) (see Fig. 1B) provided an $\sim 27 \mathrm{~nm}$ red-shift. The direction of the LSPR shift is in agreement with an increase in local R.I. $(n=1.5)$ of Au TNPs due to formation of the SAM. We selected DDT as part of the SAM because it would not only act as a spacer and reduce steric hindrance between proteins, but it would also avoid nonspecific adsorption of unwanted biomolecules onto the surface of TNPs. Secondly, the SAM-modified Au TNPs were then reacted with $0.2 \mathrm{M}$ EDC/NHS solution to active the acid group of MHDA followed by incubation in anti-cTnT overnight (Fig. 1C), which resulted in an additional $12 \mathrm{~nm}$ red-shift of the $\lambda_{\text {LSPR }}$ (see Fig. $2 \mathbf{E}$ and insert red double headed arrow). Attachment of anti-cTnT produced our chip-based format LSPR cTnT biosensor. We selected chemical attachment of anti-cTnT to Au TNPs through an amide bond to enhance stability and increase reproducibility by lowering loss of protein during various incubation steps. Thirdly, incubation of the biosensors in cTnT solution (Fig. 1D) should provide an additional red-shift in $\lambda_{\text {LSPR }}($ Fig. 1E) as a consequence of the change in the local R.I. that occurred from cTnT binding to anti-cTnT-cTnT. As shown in Fig. 2E (insert black double headed arrow), this hypothesis is in agreement with an $\sim 7 \mathrm{~nm}$ red-shift of $\lambda_{\text {LSPR }}$ upon incubation of biosensors in $10 \mathrm{nM}$ (350,000 ng/L) cTnT solution in PBS buffer. Finally, concentration-dependent $\lambda_{\text {LSPR }} \operatorname{shifts}\left(\Delta \lambda_{\text {LSPR }}\right)$ of the biosensors before and after cTnT incubation were calculated from the center of the LSPR peak, which was used to determine LODs (Fig. 1E). We also determined the step-wise $\lambda_{\text {LSPR }}$ shifts of the other two edge-length Au TNPs (Fig. 2F). We indeed observed that the largest edge-length $\mathrm{Au}$ TNPs displayed the highest shift as a consequence of their highest sensing volume. 
Table S1 and S2 summarize the LODs of our LSPR cTnT biosensors fabricated with 34, 42, and 47 nm edge-length Au TNPs, which were determined to be 974, 75.4, and $0.9 \mathrm{ng} / \mathrm{L}$, respectively. A detail procedure for LOD calculation is provided in the Experimental Section. Our LOD values for cTnT (protein) detection are in agreement with the literature report in which gold nanoparticles with the largest diameter displayed the highest LSPR sensing ability for detection of streptavidine (protein) in PBS buffer. ${ }^{21}$ Importantly, the concentration-dependent $\lambda_{\text {LSPR }}$ shifts are found to be linear at lower cTnT concentration (Fig. 3). The linear detection ranges of cTnT for 47, 42, and $34 \mathrm{~nm}$ edge-length $\mathrm{Au}$ TNPs were 3.5-35000, 350-35000, and 350-35000 ng/L, respectively. Our finding of a 3-4 order of magnitude linear range for cTnT detection corroborates literature report on LSPR-based detection of protein (antibody). ${ }^{48}$ Furthermore, the error bars are smaller at lower concentration, indicating better quantifying ability of our biosensors at lower concentration range. These experimental values are critical in terms of detecting low abundance biomolecules, which has been found challenging using LSPR biosensors. ${ }^{49}$ Nevertheless, the lowest LOD of $0.9 \mathrm{ng} / \mathrm{L}$ (26 $\mathrm{fM}$ ) is $\sim 13$ fold better than the commercial instrument, which is commonly used to quantify cTnT levels in patients with cardiovascular diseases. ${ }^{18}$ It is important to mention that we did not consider the effect of potential plasmonic coupling between the adjacent Au TNPs that could enhance the overall sensitivity.

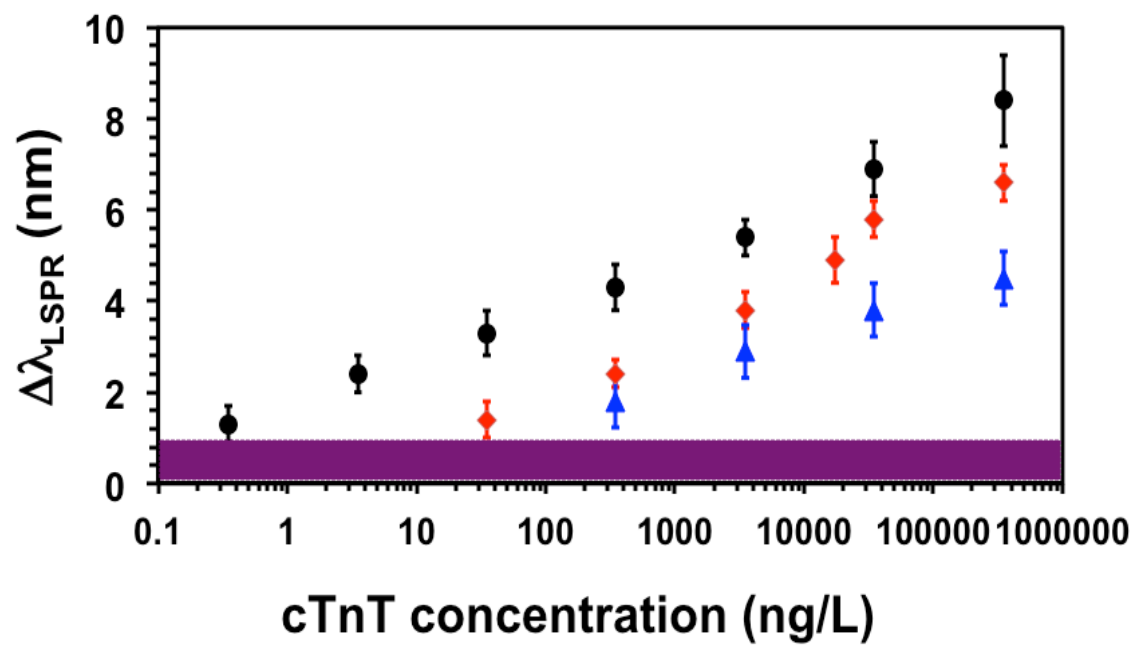

Fig. 3: Determination of sensing efficiency of LSPR cTnT biosensors. Plot of average $\Delta \lambda_{\mathrm{LSPR}}$ of biosensors that were fabricated with three different edge length Au TNPs as a function of cTnT concentration (in logarithm) in PBS buffer: black dots (47 nm edge-length), red diamonds (42 nm edge-length), and blue triangles (34 nm edge-length). The purple bar represents three times the standard deviation ( $\sigma$ ) of the blank (mixed anti-cTnT/DDT functionalized Au TNPs attached onto a glass coverslip). The concentration was 
plotted in log scale to examine the non-specific adsorption of proteins at the lower concentration range, as is routinely used to characterize the sensitivity of the LSPR biosensors. ${ }^{21,50}$

Our LSPR cTnT biosensors with the best sensitivity were prepared with $47 \mathrm{~nm}$ edge-length Au TNPs and displayed an LOD of $26 \mathrm{fM}$ in PBS buffer. This is in agreement with the literature that the largest nanostructures display the highest sensitivity because of their largest sensing volume. However, the various required surface functionalizations in the process of sensors fabrication for this edge-length $\mathrm{Au}$ TNPs resulted a $\lambda_{\text {LSPR }}$ peak in the near-infrared region at $\square 910 \mathrm{~nm}$ (see Figure S2). Here the water absorption peak can potentially interfere with the $\lambda_{\text {LSPR }}$ maximum of Au TNPS and cause misleading LOD values. To avoid potential challenges in precise quantification of cTnT in complex biological fluids using our biosensors, we decided to use $42 \mathrm{~nm}$ edge length Au TNPs $(\mathrm{LOD}=2.2 \mathrm{pM})$ for further investigation of the effects of decay length.

Standardization of Surface Chemistry and Decay Length of LSPR cTnT Biosensors. The equation below reported by Campbell and coworkers to quantify the overall response of surface plasmon resonance sensors ${ }^{51}$ is commonly used to characterize the sensitivity of LSPR biosensors: ${ }^{13}$

$$
\Delta \lambda_{L S P R}=m \Delta n e^{-2 r / L}\left(1-e^{-2 r / L}\right)
$$

Here $\Delta \mathrm{n}$ is the change in refractive index caused by the analyte adsorption, $\mathrm{m}$ is the refractive index sensitivity, $\mathrm{r}$ is the distance between the nanostructure surface and analyte, and $\mathrm{L}$ is the EMfield decay length. This equation shows that $\Delta \lambda_{\text {LSPR }}$ decreases exponentially as $r$ increases because of the exponential decrease of the EM-field. According to our previous experimental determination, the EM-field decay length of 34, 42, and $47 \mathrm{~nm}$ edge-length Au TNPs are 22, 24, and $26 \mathrm{~nm}$, respectively. ${ }^{42}$ Therefore, we hypothesize that the sensitivity of our LSPR-based cTnT biosensor will increase as distance between the Au TNP and anti-cTnT receptor decreases, which can be achieved by shorting the chain length of the alkylthiols used in the SAM. To validate our hypothesis, as described before, we selected MUDA/NT and MHNA/HT SAMs to fabricate our biosensors. We followed the same fabrication strategy as described for the MHDA/DDT SAM. 


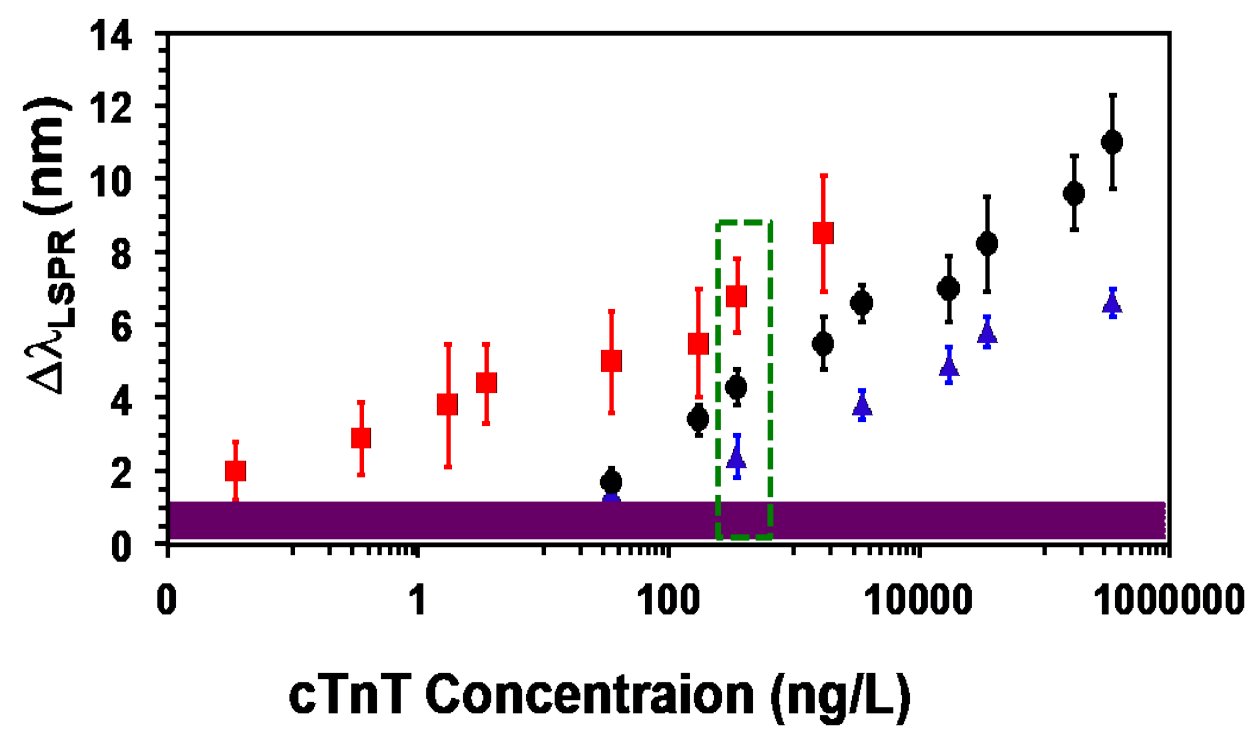

Fig. 4. Determination of efficient decay length of LSPR cTnT biosensors. Plot of average $\Delta \lambda_{\mathrm{LSPR}}$ of the LSPR biosensors, which were fabricated with three different types of SAMs: blue triangles (MHDA/DDT), black dots (MUDA/NT), and red squares (MHNA/HT) versus the logarithm of cTnT concentration. The purple bar represents three times the standard deviation $(\sigma)$ of the blank (mixed anti-cTnT/DDT functionalized $\mathrm{Au}$ TNPs attached onto a glass coverslip). Green dotted box shows $\Delta \lambda_{\mathrm{LSPR}}$ for three different SAMmodified sensors at $350 \mathrm{ng} / \mathrm{L}$ cTnT concentration in PBS buffer. The linear detection ranges of cTnT for MHDA/DDT, MUDA/NT, and MHNA/HT SAMs were 350-35000, 35-17500, and 0.035-3.5 ng/L, respectively.

Fig. 4 shows the concentration dependent $\lambda_{\text {LSPR }}$ shifts for our cTnT biosensors that were prepared with three different spacer length SAMs. As expected, the sensor prepared with MHNA/HT SAM provided the shortest distance between the nanostructure and analyte, and produced the lowest LOD of 3 fM (see Table S3 and S4). Fig. S3 shows extinction spectra of our biosensors prepared with the three different SAMs before and after incubation in PBS buffer at $350 \mathrm{ng} / \mathrm{L} \mathrm{cTnT}$. We purposefully selected a lower concentration range for LOD determination for the sensor constructed with MHNA/HT SAM in comparison to the sensors fabricated with MUDA/NT and MHDA/DDT SAMs to evaluate the limitation of the LSPR biosensors. In general in the case of miniaturize LSPR biosensors, quantifying low concentrations of analyte is extremely challenging. Even though the cTnT sensor constructed with MHNA/HT SAM provided the best sensitivity, there are still several drawbacks associated with this sensor: 
(i) Firstly, there was a large variation between performance with different sensors as observed

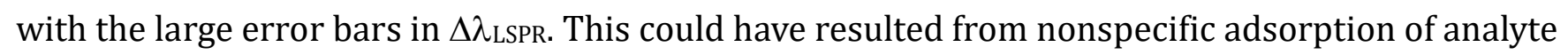
as a consequence of defects in the MHNA/HT SAMs, which contained alkylthiols with 6 methylene units. It is known that for formation of a perfectly packed SAM of alkylthiols on a gold surface (i.e., without the presence of defects or pinholes), a minimum of 9 methylene units is required..$^{52}$

(ii) Secondly, non-linearity was observed in the concentration range of 3.5 -350 ng/L (Fig. 4, red squares). This concentration range is critical because a patient with cTnT concentration $>10 \mathrm{ng} / \mathrm{L}$ can be identified potential risk for MCI. ${ }^{29,34}$

(iii) Thirdly, a shorter distance between the nanostructure and receptor will induce long-range attractive forces originating from the underlying solid substrate, which results in error in the analysis. ${ }^{53}$ Thus, a relative larger distance is required to reduce such forces.

(iv) Finally, for potential application it is extremely important to examine the selectivity of our LSPR cTnT biosensors. Therefore, we performed two important control experiments on our sensors prepared with MHNA/HT and MUDA/NT SAMs. In the first experiment, MHNA/HT and MUDA/NT SAM-modified Au TNPs attached onto silanized glass substrates were incubated over-

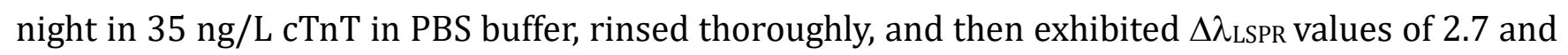
$0.4 \mathrm{~nm}$, respectively (Fig. S4 and Fig. S5). Therefore, the actual sensor (Fig. 1) design involving MHNA/HT SAM has the possibility of providing false positive responses. In contrast, the negligible shift observed for MUDA/NT SAM is within the instrument noise level. In the second control experiment, we selected cTnT sensors, which were prepared with both the above-mentioned SAMs and then incubated in $35 \mathrm{ng} / \mathrm{L}$ tropomyosin, a protein that is present in the heart muscle and is also released during a heart attack. Clearly for practical application, it is very important to study the selectivity of our chip format LSPR cTnT biosensor. Fig. S5 illustrates UV-visible extinction spectra of our sensors constructed with two different SAMs before and after tropomyosin incubation. The sensor constructed with MUDA/NT SAM displayed no noticeable $\lambda_{\text {LSPR }}$ shift, whereas an $\sim 4 \mathrm{~nm} \Delta \lambda_{\text {LSPR }}$ was observed with the sensor constructed with MHNA/HT SAM, indicating a nonspecific response of the sensor in the later case. We should mention that this $4 \mathrm{~nm}$ shift corresponds to a concentration of $2.4 \mathrm{ng} / \mathrm{L}$ (see Table S3), which is within the concentration range of patients with the risk of a heart attack. Based on our experimental data and literature reports, our LSPR biosensor constructed with MUDA/NT SAMs is more capable of avoiding false positive responses in terms of nonspecific adsorption of an important unwanted analyte on the sensors sur- 
face. Thus, we selected MUDA/NT SAM for chip-based fabrication of our LSPR cTnT biosensor as part of our further investigation.

Enhancement of cTnT Binding onto LSPR cTnT Biosensors. In addition to the sensing volume and decay length of metal nanostructures, the number of receptor sites present on the LSPR biosensor directly influences the $\lambda_{\text {LSPR }}$ shift, as reported by Chilkoti and coworkers for Au nanorods as described by Eq. 2.43

$$
\Delta \lambda_{L S P R}=\frac{3 S_{0} e^{-2 r / L}}{V_{S}} \Delta R I \cdot N \cdot V_{A}
$$

Here $S_{0}$ is bulk R.I. sensitivity, $\Delta R I$ is the difference in R.I. between the analyte and the medium, and $\mathrm{N}$ and $\mathrm{V}_{\mathrm{A}}$ are the number of molecules bound to the sensor and volume of the analyte molecule, respectively.

Using Eq. 2 we hypothesize that by increasing the number of cTnT binding sites while not causing steric and/or electrostatic repulsion between anti-cTnT molecules and thus decreasing nonspecific analytes adsorption, we will be able to increase the sensitivity of our LSPR cTnT sensor. To validate our hypothesis, we selected $42 \mathrm{~nm}$ edge-length Au TNPs and MUDA/NT SAMs to keep sensing volume and decay length, respectively, to their optimum values and best selectivity for chip-based cTnT biosensor fabrication as described above. Then $\mathrm{N}$ was varied by changing the ratio of MUDA:NT (Eq. 2). We expect that an increase of MUDA concentration on the Au TNP surface would increase the number of receptor anti-cTnT sites on the sensor and thus more cTnT would bind, resulting in larger $\lambda_{\text {LSPR }}$ shifts. Fig. $\mathbf{S 6}$ shows the LSPR shift of our sensor before and after incubation in $1750 \mathrm{ng} / \mathrm{L}$ cTnT in PBS buffer. Table S5 summarizes the average $\Delta \lambda_{\mathrm{LSPR}}$ value for four different ratios. The highest $\Delta \lambda_{\text {LSPR }}$ we observed of $11.5 \mathrm{~nm}$ for 8:2 MUDA/NT is in agreement with our hypothesis and Eq. 2. We did not investigate the sensor responses for 100\% MUDA SAM because the presence of a spacer in SAMs is extremely important in reducing repulsion between the receptor sites and avoiding the non-specific adsorption of analytes in LSPR biosensors, as reported in the literature. ${ }^{10,43,49}$ Therefore, we determined that the best nanoscale structural parameters for chip-based fabrication of our LSPR cTnT biosensor are: (i) an 42 nm edge-length Au TNP optimization of sensing volume, (ii) $1.7 \mathrm{~nm}$ distance between Au TNP and anti-cTnT - standardization of decay length (MUDA/NT SAMs), and (iii) an 8:2 ratio of receptor-to-spacer - enhancement 
of cTnT binding (MUDA:NT SAMs). Based on these optimized parameters, we determined the LOD of cTnT in PBS buffer and found it to be 7.2 aM (Fig. 5A). To the best of our knowledge, this is the best sensitivity for cTnT detection reported in the literature. ${ }^{19}$ More importantly, our LOD is nearly 50 times better than recently reported for label-free detection of cTnT using a diode-based electrochemical technique, which requires a complex fabrication strategy. ${ }^{19}$ As shown in Fig. 5B, we calculated the $\mathrm{K}_{\mathrm{D}}$ value by fitting the data to a Langmuir isotherm and found it to be $4.23 \times 10^{-7} \mathrm{M}$ in PBS buffer. This result suggests that the target protein cTnT has relative strong affinity toward its antibody. Under our experimental conditions where the LOD reaches to few tens of aM, the cTnTs can easily detach from the sensors surface because of their relatively high KD value. This could be a potential reason from the deviation of linearity at such very low concentration. Surprisingly, even though the $\mathrm{K}_{\mathrm{D}}$ value is relatively high for cTnT, the ultrasensitive nature of our LSPR biosensor, which was constructed with appropriate optimization of structural parameters, allowed us to assay concentration as low as $3.5 \times 10^{-4} \mathrm{ng} / \mathrm{L}$ with a LOD of $251 \times 10^{-6} \mathrm{ng} / \mathrm{L}$ in PBS buffer.
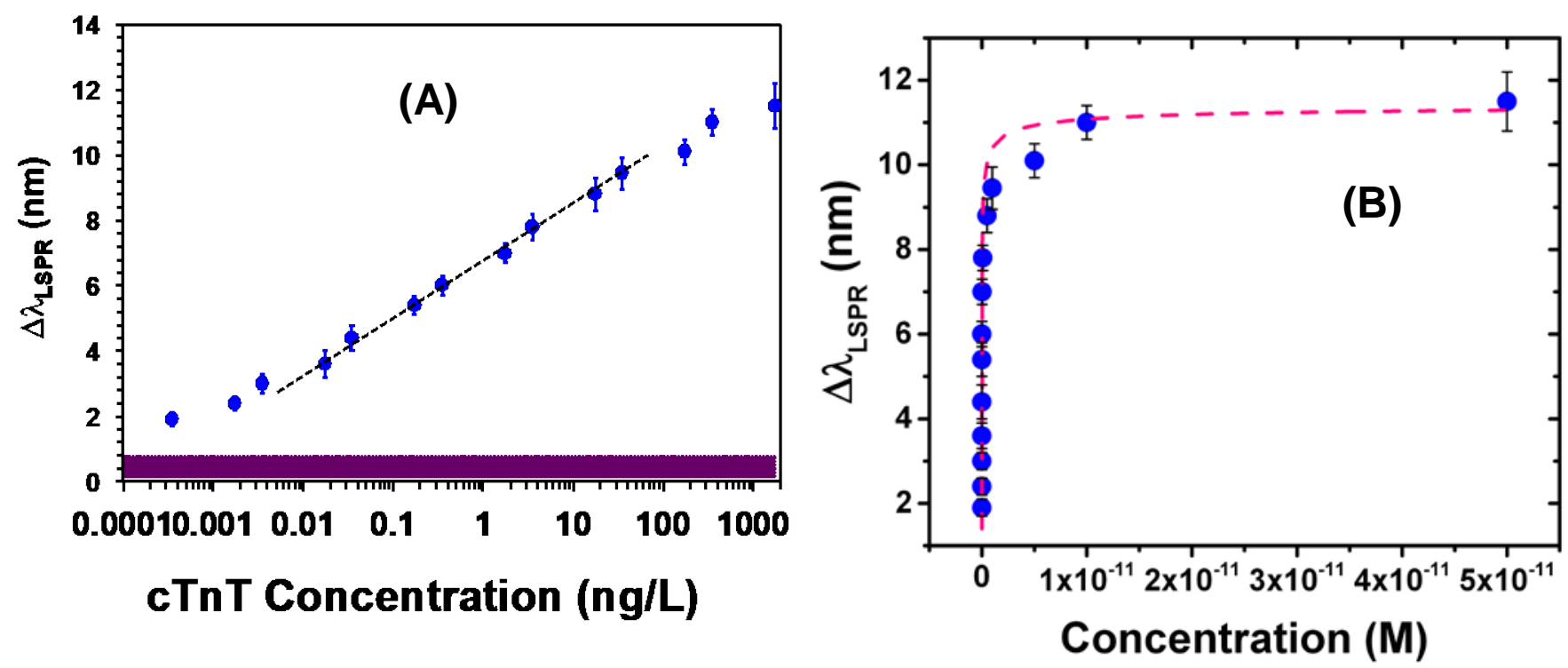

Fig. 5. Determination of Sensing Efficiency of LSPR cTnT biosensors. (A) Plot of average $\Delta \lambda_{\mathrm{LSPR}}$ of the LSPR biosensors that were fabricated with $42 \mathrm{~nm}$ edge length Au TNPs functionalized with 8:2 mole ratio of MUDA:NT SAMs versus the logarithm of cTnT concentration. The dotted black line shows the linear concentration range that is within the level identified for patients at high risk for heart attack.29,34 The purple bar represents three times the standard deviation $(\sigma)$ of the blank (mixed anti-cTnT/NT-functionalized Au 
TNPs attached onto a glass coverslip). (B) Binding of cTnT to LSPR biosensor in PBS buffer. The data were fitted to a Langmuir isotherm (dotted line) to determine the $K_{D}$ value.

\section{Potential applicability of chip-based LSPR cTnT biosensor for point-of-care diagnostics.}

Over the last 10 years there has been a growing interest in LSPR-based assays as label-free, low cost medical diagnostic tools. To meet the needs not only of point-of-care diagnostics but also potential laboratory applications, it is important that the LSPR biosensors be able to assay analytes in complex biofluids, such as plasma, serum, urine, etc. With the aim of potential biomedical applications, we examined the working capability of our chip-based LSPR cTnT biosensor in undiluted human plasma and serum, and 50\% human urine. We prepared a cTnT stock solution using these biological fluids and then lowered the cTnT concentrations through a series of dilutions with the respective biological fluids. The sensor was fabricated using our optimized parameters as described for PBS buffer. Fig. 6A illustrates concentration-dependent average $\Delta \lambda_{\text {LSPR }}$ value of our cTnT biosensors in the three different fluids. The LODs in plasma and serum were determined to be $\sim 14$ and $22 \mathrm{aM}$, respectively, where as the LOD value is slightly higher (1.9 fM) in urine (see Table S6 and Table S7).
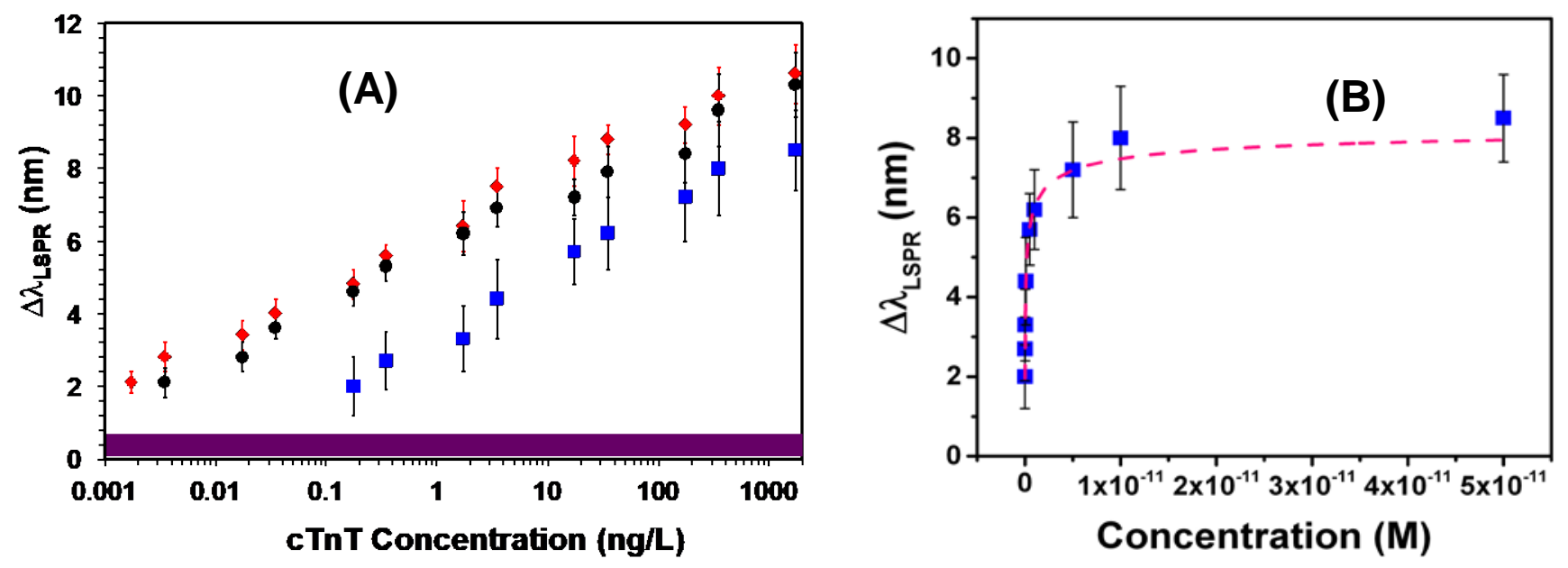

Fig. 6. Assaying cTnT in human biofluids. (A) Plot of average $\Delta \lambda_{\text {LSPR }}$ of the chip-based format cTnT biosensors in undiluted plasma (red diamonds), serum (black dots), and 50\% urine (blue squares) versus the logarithm of cTnT concentration. Urine samples were diluted with PBS buffer. (B) Binding of cTnT to biosensor in 50\% urine. The data were fitted to a Langmuir isotherm (dot- 
ted line) to determine the $\mathrm{K}_{\mathrm{D}}$ value of $1.1 \times 10^{-5} \mathrm{M}$. The linear detection ranges of cTnT for plasma, serum, and urine were 0.0175-35, 0.0035-3.5, and 3.5-350 ng/L, respectively.

Considering that tens of thousand of proteins are present in these complex human biofluids, our results, specifically the sensitivity and selectivity of the cTnT assay in human plasma and serum, is outstanding. Strikingly, the LOD we determined in undiluted plasma ( $14 \mathrm{aM})$ is more than $10^{3}$ times better than that the LOD reported for cTnT detection of $\sim 300 \mathrm{fM}$ using a microfluidic diodebased device monitoring current-voltage response in undiluted human serum. ${ }^{19}$ Furthermore, our sensor in plasma displays $4 \times 10^{3}$ times better LOD than the electrochemicaly determined cTnT concentration involving a ZnO nanostructure. ${ }^{20}$ Table S8 shows a comparison of LODs reported for different cTnT detection methods. Importantly, to the best of our knowledge, our label-free LSPR-based cTnT assay displayed the lowest LOD in the literature. This highlights the unique advantage of designing and utilizing LSPR biosensors to assay disease biomarkers with an unprecedentedly low LOD. Surprisingly, we observed a high value of LOD and large variation of $\Delta \lambda_{\text {LSPR val- }}$ ues in urine. One would expect better sensitivity of the LSPR biosensors in urine in comparison to either plasma and/or serum because of a relatively lower concentration of proteins, thus reducing nonspecific binding onto the sensor surface. We believe that the high urea concentration in urine screens the interaction between anti-cTnT and cTnT, resulting in low sensitivity. The calculated high $K_{D}$ value of cTnT in urine of $1.1 \times 10^{-5} \mathrm{M}$ (see Fig. 6B) shows nearly 100 fold less affinity toward its antibody in comparison to the other two human biofluids (Fig. S7).

\section{CONCLUSIONS}

In conclusion, our systematic study unravels the effects of various nanoscale parameters, which modulate the overall sensitivity of our chip-based format LSPR biosensor. This plasmonic nanosensor uses LSPR shifts to quantitate an important disease biomarker without complicated fabrication strategies. Our sensor displayed LOD as low as $\sim 250 \times 10^{-6} \mathrm{ng} / \mathrm{L}$ in untreated human plasma, and also showed feasibility of working in other human biofuilds (serum and urine) in a concentration range much lower than that of the commercial ECLIA instrument from Roche Diagnostic. ${ }^{18}$ Importantly, development of our LSPR-based assay provides several guiding principles in the fabrication of optical-based biosensors: Firstly, longer edge length (larger sensing volume) $\mathrm{Au}$ TNPs display the highest sensitivity for cTnT detection, however their near infra-red LSPR peak position could potentially be interfered with by overlapping with the absorption peaks of water 
and other biomolecules. Thus, metal nanostructures with LSPR peaks in the near infrared region should be avoided for sensor fabrication. Secondly, appropriate selection of SAM length, which connects receptor and nanostructure and positions the anti-cTnT at the appropriate EM-field decay length is also critical to reducing non-specific adsorption of biomolecules on the surface of sensor while maintain the highest sensitivity. Additionally, increasing the number of receptor molecules on the surface of $\mathrm{Au}$ TNPs while reducing the steric and/or electrostatic repulsion between analytes is critical to achieving highest sensitivity. Taken together, we have determined that the following structural parameters are the best combination for assaying cTnT: (i) an $\sim 42 \mathrm{~nm}$ edgelength $\mathrm{Au}$ TNP, (ii) $1.7 \mathrm{~nm}$ distance between Au TNP and anti-cTnT using MUDA/NT SAMs, and (iii) an 8:2 ratio of receptor-to-spacer. We believe that our work on control manipulation of surface functionalization and immobilization of bio-recognition molecules will provide an experimental benchmark for the fabrication of chip-based format, ultrasensitive nanoplasmonic biosensors. Finally, we have demonstrated excellent reproducibility (batch-to-batch variation) of our sensor fabrication technique, and thus it has the potential for chip-based technology development for practical applications for early diagnosis of heart attack. ${ }^{29,34}$ Though, our bioseneors demonstrate exceptional LOD for cTnT detection but this sensor may not be able to quantitatively determine cTnT concentration from a single cell extracts.

\section{Electronic Supplementary Information}

Experimental procedure, analytical techniques, additional extinction spectra, tables, calibration plots, Langmuir isotherms, and additional discussion relevant to reproducibility of sensor fabrication. This material is available free of charge via the Internet at http://pubs.acs.org.

\section{ACKNOWLEDGMENT}

This work was funded through the National Science Foundation (CBET-1604617). We thank Prof. B. Muhoberac, Dr. A. Siegel, and Ms. S. Savant for helpful suggestions.

\section{REFERENCES}

1 Jain, P. K.,Huang, X.,El-Sayed, I. H. and El-Sayed, M. A. Acc. Chem. Res., 2008, 41, 15781586.

2 Guerreiro, J. R. L.,Frederiksen, M.,Bochenkov, V. E.,De Freitas, V.,Ferreira Sales, M. G. and Sutherland, D. S. ACS Nano, 2014, 8, 7958-7967.

3 Otte, M. A.,Sepulveda, B.,Ni, W.,Juste, J. P.,Liz-Marzan, L. M. and Lechuga, L. M. ACS Nano, 2009, 4, 349-357.

$4 \quad$ Willets, K. A. and Van Duyne, R. P. Ann. Rev. Phys. Chem., 2007, 58, 267-297.

5 Halas, N. J.,Lal, S.,Chang, W.-S.,Link, S. and Nordlander, P. Chem. Rev., 2011, 111, 39133961.

6 Mayer, K. M. and Hafner, J. H. Chem. Rev., 2011, 111, 3828-3857.

7 Saha, K.,Agasti, S. S.,Kim, C.,Li, X. and Rotello, V. M. Chem. Rev., 2012, 112, 2739-2779. 
Stewart, M. E.,Anderton, C. R.,Thompson, L. B.,Maria, J.,Gray, S. K.,Rogers, J. A. and Nuzzo, R. G. Chem. Rev., 2008, 108, 494-521.

9 Haes, A. J.,Chang, L.,Klein, W. L. and Van Duyne, R. P. J. Am. Chem. Soc., 2005, 127, 22642271.

10 Haes, A. J.,Zou, S.,Zhao, J.,Schatz, G. C. and Van Duyne, R. P. J. Am. Chem. Soc., 2006, 128, 10905-10914.

11 Ahijado-Guzmán, R.,Prasad, J.,Rosman, C.,Henkel, A.,Tome, L.,Schneider, D.,Rivas, G. and Sönnichsen, C. Nano Lett., 2014, 14, 5528-5532.

12 Haes, A. J.,Hall, W. P.,Chang, L.,Klein, W. L. and Van Duyne, R. P. Nano Lett., 2004, 4, 10291034.

13 Anker, J. N.,Hall, W. P.,Lyandres, O.,Shah, N. C.,Zhao, J. and Van Duyne, R. P. Nat. Mater., 2008, 7, 442-453.

14 Fong, K. E. and Yung, L.-Y. L. Nanoscale, 2013, 5, 12043-12071.

15 Joshi, G. K.,Deitz-McElyea, S.,Liyanage, T.,Lawrence, K.,Mali, S.,Sardar, R. and Korc, M. ACS Nano, 2015, 9, 11075-11089.

16 Joshi, G. K.,Deitz-McElyea, S.,Johnson, M.,Mali, S.,Korc, M. and Sardar, R. Nano Lett., 2014, 14, 6955-6963.

17 Mock, J. J.,Smith, D. R. and Schultz, S. Nano Lett., 2003, 3, 485-491.

18 Katus, H. A. Clin. Chem., 2008, 54, 1576-1577.

19 Liu, Y. and Yobas, L. Nano Lett., 2014, 14, 6983-6990.

20 Shanmugam, N. R.,Muthukumar, S. and Prasad, S. Sci. Rep., 2016, 6, 33423.

21 Nath, N. and Chilkoti, A. Anal. Chem., 2004, 76, 5370-5378.

22 Vollmer, F.,Braun, D.,Libchaber, A.,Khoshsima, M.,Teraoka, I. and Arnold, S. Appl. Phys. Lett., 2002, 80, 4057-4059.

23 Stenberg, E.,Persson, B.,Roos, H. and Urbaniczky, C. J. Colloid Interface Sci., 1991, 143, 513526.

24 El-Sayed, M. A. Acc. Chem. Res., 2001, 34, 257-264.

25 Kedem, O.,Vaskevich, A. and Rubinstein, I. J. Phys. Chem. C, 2014, 118, 8227-8244.

26 Blich, M.,Sebbag, A.,Attias, J.,Aronson, D. and Markiewicz, W. Am. J. Cardio., 2008, 101, 1384-1388.

27 Wright, R. S.,Williams, B. A.,Cramner, H.,Gallahue, F.,Willmore, T.,Lewis, L.,Ladenson, J. H. and Jaffe, A. S. Am. J. Cardio., 2002, 90, 634-636.

28 Roongsritong, C.,Warraich, I. and Bradley, C. Chest, 2004, 125, 1877-1884.

29 Wang, T. J. Circulation, 2007, 116, 1217-1220.

30 Søyseth, V.,Bhatnagar, R.,Holmedahl, N. H.,Neukamm, A.,Høiseth, A. D.,Hagve, T.-A.,Einvik, G. and Omland, T. Heart, 2013, 99, 122-126.

31 Mehta, N. J.,Khan, I. A.,Gupta, V.,Jani, K.,Gowda, R. M. and Smith, P. R. Int. J. Cardio., 2004, 95, 13-17.

32 Ammann, P.,Maggiorini, M.,Bertel, O.,Haenseler, E.,Joller-Jemelka, H. I.,Oechslin, E.,Minder, E. I.,Rickli, H. and Fehr, T. J. Am. Coll. Cardio., 2003, 41, 2004-2009.

33 Ndumele, C. E.,Coresh, J.,Lazo, M.,Hoogeveen, R. C.,Blumenthal, R. S.,Folsom, A. R.,Selvin, E.,Ballantyne, C. M. and Nambi, V. JACC: Heart Failure, 2014, 2, 600-607.

34 Omland, T.,de Lemos, J. A.,Sabatine, M. S.,Christophi, C. A.,Rice, M. M.,Jablonski, K. A.,Tjora, S.,Domanski, M. J.,Gersh, B. J.,Rouleau, J. L.,Pfeffer, M. A. and Braunwald, E. New Engl. J. Med., 2009, 361, 2538-2547.

35 Wong, P.,Murray, S.,Ramsewak, A.,Robinson, A.,van Heyningen, C. and Rodrigues, E. Postgrad. Med. J., 2007, 83, 200-205.

36 Hassan, A. K. M.,Bergheanu, S. C.,Hasan-Ali, H.,Liem, S. S.,van der Laarse, A.,Wolterbeek, R.,Atsma, D. E.,Schalij, M. J. and Jukema, J. W. Am. J. Cardiol., 103, 779-784. 
37 Guo, L.,Jackman, J. A.,YYang, H.-H.,Chen, P.,Cho, N.-J. and Kim, D.-H. Nano Today, 2015, 10, 213-239.

38 Soares, L.,Csaki, A.,Jatschka, J.,Fritzsche, W.,Flores, O.,Franco, R. and Pereira, E. Analyst, 2014, 139, 4964-4973.

39 Hao, E. and Schatz, G. C. J. Chem. Phys., 2004, 120, 357-366.

40 Chien, M.-H.,Nien, L.-W.,Chao, B.-K.,Li, J.-H. and Hsueh, C.-H. Nanoscale, 2016, 8, 36603670.

41 Nehl, C. L.,Liao, H. and Hafner, J. H. Nano Lett., 2006, 6, 683-688.

42 Joshi, G. K.,McClory, P. J.,Muhoberac, B. B.,Kumbhar, A.,Smith, K. A. and Sardar, R. J. Phys. Chem. C, 2012, 116, 20990-21000.

43 Nusz, G. J.,Curry, A. C.,Marinakos, S. M.,Wax, A. and Chilkoti, A. ACS Nano, 2009, 3, 795806.

44 Joshi, G. K.,Blodgett, K. N.,Muhoberac, B. B.,Johnson, M. A.,Smith, K. A. and Sardar, R. Nano Lett., 2014, 14, 532-540.

45 Joshi, G. K.,Smith, K. A.,Johnson, M. A. and Sardar, R. J. Phys. Chem. C, 2013, 117, 2622826237.

46 Joshi, G. K.,White, S. L.,Johnson, M. A.,Sardar, R. and Jain, P. K. J. Phys. Chem. C, 2016, 120, 24973-24981.

47 Joshi, G. K.,Johnson, M. A. and Sardar, R. RSC Advances, 2014, 4, 15807-15815.

48 Endo, T.,Kerman, K.,Nagatani, N.,Hiepa, H. M.,Kim, D.-K.,Yonezawa, Y.,Nakano, K. and Tamiya, E. Anal. Chem., 2006, 78, 6465-6475.

49 Feuz, L.,Jonsson, M. P. and Höök, F. Nano Lett., 2012, 12, 873-879.

50 Marinakos, S. M.,Chen, S. and Chilkoti, A. Anal. Chem., 2007, 79, 5278-5283.

51 Jung, L. S.,Campbell, C. T.,Chinowsky, T. M.,Mar, M. N. and Yee, S. S. Langmuir, 1998, 14, 5636-5648.

52 Love, J. C.,Estroff, L. A.,Kriebel, J. K.,Nuzzo, R. G. and Whitesides, G. M. Chem. Rev., 2005, 105, 1103-1170.

53 Feuz, L.,Jönsson, P.,Jonsson, M. P. and Höök, F. ACS Nano, 2010, 4, 2167-2177. 